\title{
Prefix for Binary Multiples
}

National Cancer Institute

\section{Source}

National Cancer Institute. Prefix for Binary Multiples. NCI Thesaurus. Code C68560.

A prefix used in binary numeral system for denoting powers of two. 